\title{
Umbilical endometriosis in a patient without abdominal surgery
}

\begin{abstract}
Endometriosis is the presence of endometrial glands and stroma outside the uterine cavity. It is an estrogen-dependent disease. Can appear anywhere in the body, most commonly in the ovaries, anterior and posterior douglas pounch, posterior broad ligaments, uterosacral ligaments, fallopian tubes, sigmoid colon, appendix and round ligaments. In women with previous C-section, umbilical endometriosis can occur in $30 \%$ of cases. It is a common nonmalignant cause of dysmenorrhea, dyspareunia, chronic pain, and infertility. The symptoms depend on the implantation of the ectopic stroma. The prevalence depends if women are asymptomatic (1-7\%), or symptomatic that is much higher and variable ( $70 \%$ in women and adolescents with pelvic pain, $50 \%$ in infertile women and $40 \%$ of adolescents with genital tract anomalies)
\end{abstract}

Keywords: umbilical endometriosis, abdominal pain
Volume 9 Issue 5 - 2018

\author{
Darlene Adolfo,' Pedro Brandão, ${ }^{2}$ Paula \\ Ramôa, ${ }^{3}$ Amelia Almeida ${ }^{4}$ \\ 'Department of Interne Medicine, Hospital Sousa Martins, \\ Portugal \\ ${ }^{2}$ Department of Gynecologic/Obstetric, Centro Hospitalar \\ Tâmega e Sousa, Portugal \\ ${ }^{3}$ Department of Gynecologic/Obstetric, Hospital Lusíadas, \\ Portugal \\ ${ }^{4}$ Department of Gynecologic/Obstetric, Centro Hospitalar \\ Médio Ave, Portugal
}

Correspondence: Darlene Adolfo, Department of Interne Medicine, Hospital Sousa Martins, Guarda, Portugal,Tel 0035964598 I 14, Email darlene2@lior.com.pt

Received: July 30, 2018 | Published: September 18, 2018

\section{Introduction}

Sampson in 1927 described the endometriosis as the presence of endometrial glands and stroma outside the uterine cavity and formulated the theory of transplantation. There are other 5 theories that try to explain the etiology of the endometriosis. ${ }^{1-3}$

It's an estrogen-dependent disease that can appear anywhere in the body, most commonly in peritoneum, the ovaries, anterior and posterior douglas pounch, posterior broad ligaments, uterosacral ligaments, fallopian tubes, sigmoid colon, appendix and round ligaments, less common in lungs, thorax, brain and pericardium. In women with previous $\mathrm{C}$-section, umbilical endometriosis can occur in $30 \%$ of cases. ${ }^{1-5}$

Endometriosis is a common nonmalignant cause of dysmenorrhea, dyspareunia, chronic pain, and infertility. The diagnosis based in symptoms is difficult; it can vary from complete absence of symptoms to disabling pelvic pain during the menstrual period and infertility. The prevalence depends if women are asymptomatic (1-7\%), or symptomatic that is much higher and variable $(70 \%$ in women and adolescents with pelvic pain, $50 \%$ in infertile women and $40 \%$ of adolescents with genital tract anomalies). . $^{2,3,5}$

\section{Case report}

African female patient, 33 years old, clinical antecedents unremarkable, G0P0A0, dysmenorrhea and methroragy since menarche at age 14 years old.

She was admitted in Emergency Room with an abdominal pain. She referred an umbilicus tumor, which appeared 6 months ago. It has become bigger during menstrual period, with abdominal tenderness. The pain was relieved with oral analgesic (Tramadol) (Figure 1).

In objective exam, she had a tender umbilical tumor with $2 \mathrm{~cm}$. In the ultrasound the tumor had imaging features of an umbilical abscess. The surgeon tried to drainage it but was unsuccessful.
Fourteen days after she was observed in another hospital. The observation in general surgery appointment was overlapping. The surgeon recommended removal of the umbilical tumor and scheduled the surgery. The procedure was performed 7 days after and was removed a tumor with $20 \times 20 \mathrm{~mm}$. The histology revealed an endometriosis lesion.
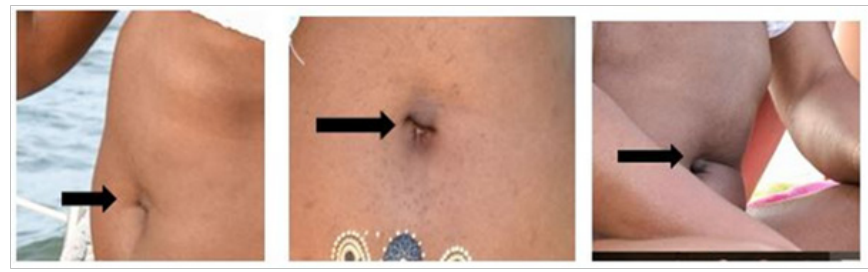

Figure I Umbilicus endometriosis.

She was forwarded for gynecologic department. In gynecologic evaluation it was found absent of uterine mobility, retroverted uterus, and uterine motion tenderness in pelvic examination. The douglas pounch was tender and a mass filling it.

Pelvic Ultrasound showed cists in the both ovaries and a kissing ovaries. In pelvic MRI was observed deep endometriosis in posterior slope of the right ovary, in Douglas pounch and in both utero sacral ligaments. It was observed many fibromas, the largest of which is in the posterior portion of the uterine body with a maximum diameter of $23 \mathrm{~mm}$. Ca 125 was $51.7 \mathrm{U} / \mathrm{mL}$

Four months later, the patient was undergone a laparoscopic surgery for endometriosis. It was found deep endometriosis in utero sacral ligaments (bilateral), in rectovaginal septum and in left ureter. Both ovaries had endometriotic cists. The uterus had multiples fibroids. The excision of the lesions of endometriosis in utero sacral ligaments and rectovaginal septum was performed. The endometrioma of both ovaries were removed. Ureteriolysis was done in left side. Some fibroids were removed. 
She was discharged the hospital after 5 days, medicated with Desogestrel 75 mycrograms. The patient was asymptomatic almost all the time.

After 14 months, she realized another pelvic MR that revealed endometriosis in abdominal subcutaneous tissue. Presence of deep endometriosis in the Douglas pounch with $8 \mathrm{~mm}$ and in the posterior vaginal wall with $5 \mathrm{~mm}$. The right ovary had presence of an endometrioma $11 * 3 \mathrm{~mm}$ and another endometrioma in the left ovary have $18 \mathrm{~mm}$. Two uterine fibroids persist.

\section{Discussion}

Endometriosis is a complex and mysterious disease for women and relatives. It is considered one of the mainly causes of gynecological hospitalization and approximately $30 \%-50 \%$ of women with the disease can develop infertility. ${ }^{3}$

According to the bibliography, cutaneous endometriosis represents $1 \%$ of all ectopic endometrium. It's classified as primary, without history of surgery, the pathogenesis is unknown. Secondary related mostly to a prior surgical scar tissue after abdominal operations - iatrogenic implantation of endometrial cells. Primary umbilical endometriosis is a rare entity, represents only $0.4 \%$ to $4.0 \%$ of all endometriosis. ${ }^{1,2,6}$

Bibliography refers that black race can be a protective factor with higher prevalence in Caucasians and Asian women. ${ }^{7}$

The natural history of the patient takes to the suspicion of diagnosis, many women from the onset of symptoms to the diagnosis take about 6 years. MRI is the key exam to determine the presence or extent of endometriosis (the experience of the Radiologist with the diseases is important, because it will determine the posture of the surgeon for the operatory moment). Serum CA-125 is a noninvasive marker, to the disease in high stages. However the gold standard continues to be the laparoscopic surgery with histologic confirmation. . $^{1,4,5,8}$

The treatment for pain can be medical: empirical analgesics, hormonal contraceptives progestogens, anti-progestagens, GnRH agonists and aromatase inhibitors - stop the evolution of endometriosis and symptoms. The surgery is an option when medical treatment fails or in some anatomic location of disease. ${ }^{1,4,9-11}$

\section{Conclusion}

With this case report the authors highlight the difficult of deep endometriosis diagnosis in absence of suspicion. In our case report the diagnosis only was made after histologic exam of endometriosis in umbilical tumor. Endometriosis continues to be an unknown pathology outside gynecologic community.

\section{Acknowledgments}

None.

\section{Conflicts of interest}

Authors did not report any potential conflicts of interests.

\section{References}

1. Sterling RK, Wright EC, Morgan TR, et al. Frequency of elevated hepatocellular carcinoma (HCC) biomarkers in patients with advanced hepatitis C. Am J Gastroenterol. 2012;107(1):64-74.

2. Yamashita T, Forgues M, Wang W, et al. EpCAM and $\alpha$-fetoprotein expression defines novel prognostic subtypes of hepatocellular carcinoma. Cancer Res. 2008;68(5):1451-1461.
3. Bruix J, Sherman M, American Association for the Study of Liver Diseases. Management of hepatocellular carcinoma: an update. Hepatology. 2011;53(3):1020-1022.

4. Tandon P, Garcia-Tsao G. Prognostic indicators in hepatocellular carcinoma: a systematic review of 72 studies. Liver Int. 2009;29(4):502510 .

5. Huo TI, Lee SD. Role of the model for end-stage liver disease and serum a-fetoprotein as predictors for hepatocellular carcinoma. Liver Int. 2006;26(10):1300-1301.

6. Lencioni R, Llovet JM. Modified RECIST (mRECIST) assessment for hepatocellular carcinoma. Semin Liver Dis. 2010;30(1):52-60.

7. Memon K, Kulik L, Lewandowski RJ, et al. Alpha-fetoprotein response correlates with EASL response and survival in solitary hepatocellular carcinoma treated with transarterial therapies: A subgroup analysis. $J$ Hepatol. 2012;56(5):1112-1120.

8. Yau T, Yao TJ, Chan P, et al. The significance of early alpha-fetoprotein level changes in predicting clinical and survival benefits in advanced hepatocellular carcinoma patients receiving sorafenib. Oncologist. 2011;16(9):1270-1279.

9. Kaibori M, Kubo S, Nagano H, et al. Clinicopathological features of recurrence in patients after 10-year disease-free survival following curative hepatic resection of hepatocellular carcinoma. World J Surg. 2013;37(4):820-828

10. Shiina $S$, Tateishi R, Arano $T$, et al. Radiofrequency ablation for hepatocellular carcinoma: 10-year outcome and prognostic factors. $\mathrm{Am}$ J Gastroenterol. 2012;107(4):569-577.

11. N'Kontchou G, Mahamoudi A, Aout M, et al. Radiofrequency ablation of hepatocellular carcinoma: long-term results and prognostic factors in 235 Western patients with cirrhosis. Hepatology. 2009;50(5):1475-1483.

12. Lee DH, Lee JM, Lee JY, et al. Radiofrequency ablation of hepatocellular carcinoma as first-line treatment: long-term results and prognostic factors in 162 patients with cirrhosis. Radiology. 2014;270(3):900-909.

13. Kikuchi L, Menezes M, Chagas AL, et al. Percutaneous radiofrequency ablation for early hepatocellular carcinoma: Risk factors for survival. World J Gastroenterol. 2014;20(6):1585-1593.

14. Giannini EG, Marenco S, Borgonovo G, et al. Alpha-fetoprotein has no prognostic role in small hepatocellular carcinoma identified during surveillance in compensated cirrhosis. Hepatology. 2012;56(4):13711379.

15. Pompili M, Rapaccini GL, Covino M, et al. Prognostic factors for survival in patients with compensated cirrhosis and small hepatocellular carcinoma after percutaneous ethanol injection therapy. Cancer. 2001;92(1):126-135.

16. Nomura F, Ohnishi K, Tanabe Y. Clinical features and prognosis of hepatocellular carcinoma with reference to serum alpha-fetoprotein levels. Analysis of 606 patients. Cancer. 1989;64(8):1700-1707.

17. Farinati F, Marino D, De Giorgio M, et al. Diagnostic and prognostic role of alpha-fetoprotein in hepatocellular carcinoma: both or neither? $\mathrm{Am} \mathrm{J}$ Gastroenterol. 2006;101(3):524-532.

18. Kiriyama S, Uchiyama K, Ueno M, et al. Triple positive tumor markers for hepatocellular carcinoma are useful predictors of poor survival. Ann Surg. 2011;254(6):984-991.

19. Zhang W, Luo E, Gan J, et al. Long-term survival of hepatocellular carcinoma after percutaneous radiofrequency ablation guided by ultrasound. World J Surg Oncol. 2017;15(1):122-128. 